\title{
The Effect of Kinesio Tape on Knee Pain and Quality of Life in Subjects with Knee Osteoarthritis - A Randomized Clinical Trial
}

\author{
Ali Karamitabar ${ }^{1}$, Shabnam Shahali*1, Mehdi Dadgoo', Soheil Mansour Sohani ${ }^{1}$, Arsalan Ghorbanpour ${ }^{2}$, \\ Hashem Abrisham Karzadeh ${ }^{3}$, Hasan Jaafari', Parnian Mirbehresi ${ }^{1}$
}

1. Department of Physiotherapy, School of Rehabilitation Sciences, Iran University of Medical Sciences, Tehran, Iran

2. Department of Physiotherapy, School of Rehabilitation Sciences, Tehran University of Medical Sciences, Tehran, Iran

3. Department of Orthopedics, Faculty of Medicine, Shahid Beheshti University of Medical Sciences, Tehran, Iran

\begin{tabular}{c} 
Article Info \\
\hline Received:2018/11/18; \\
Accepted:2019/03/17; \\
Published Online: 2019/03/2 \\
DOI: 10.30699/fdisj.2.1.27 \\
How to Cite This Article \\
Karamitabar A., Shahali S., \\
Dadgoo M., Mansour Sohani \\
S., Ghorbanpour A., Abrish- \\
am Karzadeh H.,Jaafari H., \\
Mirbehresi P. The Effect of \\
Kinesio Tape on Knee Pain \\
and Quality of Life in Sub- \\
jects with Knee Osteoarthri- \\
tis - A Randomized clinical \\
Trial. Function and Disability \\
Journal. 2019 (Winter). Vol:2 \\
.No:1. Pages: 27-36
\end{tabular}

Use your device to scan and read the article online

\section{ABSTRACT}

Background and Objectives: The short and long-term effects of Kinesio tape on pain and quality of life (functional status) in patients with knee osteoarthritis were investigated.

Materials and Methods: Forty-seven patients with knee osteoarthritis were randomly allocated into Kinesio tape and Sham Kinesio tape groups. The assessments were performed at the baseline, 30 minutes after the initial Kinesio tape application, 3 days after the fifth Kinesio tape application and 1 week later. The quality of life (functional status) of the patients was evaluated using the Western Ontario and McMaster Universities Osteoarthritis Scale (WOMAC). The pain level was measured using the visual analog scale (VAS).

Results: In both groups, the mean of the rest pain, activity pain score, and WOMAC score decreased, but there were no significant differences between groups for both VAS and WOMAC scores $(P>0.05)$. Also, there were no significant differences between groups during the follow up assessment.

Conclusion: According to the results, it may be concluded that there were no beneficial effects between Kinesio tape and sham tape on pain and on the quality of life in patients with knee osteoarthritis.

Keywords: Tape, Knee osteoarthritis, Pain, Quality of life, Kinesio tape.

Corresponding information:

Shabnam Shahali, Department of Physiotherapy, School of Rehabilitation Sciences, Iran University of Medical Sciences, Tehran, Iran E mail: shabnamshahali@yahoo.com

Copyright (C) 2019, Function and Disability Journal. This is an original open-access article distributed under the terms of the Creative Commons Attribution-noncommercial 4.0 International License which permits copy and redistribution of the material just in noncommercial usages with proper citation.

\section{Introduction}

Osteoarthritis is the most common form of arthritis which develops with aging. This disease causes joint degeneration and disability in the patient, and is one of the causes of joint replacement (Jongbloed, van Twist, and Swart, 2016). The prevalence of knee osteoarthritis in men and women has been reported to be around $40 \%$ to $47 \%$ respectively, and this sub- ject shows a positive growth rate every year (Roder , 2002). The knee is the most common joint affected by osteoarthritis, which is due to weight bearing and repetitive motions in this joint (Kocyigit et al., 2015).

The major problem of patients suffering from knee osteoarthritis is pain, which causes immobility and a diminished quality of life in these patients. Therefore, it can be expected that the reduction of different types of pain will be effective in enhancing the qual- 
ity of life of these patients (Jongbloed, van Twist, and Swart, 2016).

Treatments for knee osteoarthritis in mild to moderate stages include changing lifestyle, modifying daily activities, weight loss, medical interventions (including anti-inflammatory drugs and articular injections), physiotherapy interventions and the use of knee braces (Kocyigit et al., 2015).

Medical treatments such as pharmacotherapy have a number of side effects, and articular injections and surgery are considered as invasive treatments (Wageck et al., 2016). Today, the use of noninvasive therapeutic methods with fewer complications such as physiotherapy is attracting a great deal of attention.

Physiotherapy for treating these patients includes resistance exercises, proprioception exercises, electrical stimulations, long socks, acupuncture, manual therapy, bandage, and Kinesio tape (Kocyigit et al., 2015).

Kinesio tape was first invented by a Japanese chiropractor called Kenzo Kase in 1970 (Kocyigit et al. , 2015). After the First World War, the use of Kinesio tape by physiotherapists in musculoskeletal problems began to spread rapidly in the summer of 1988 (Jongbloed, van Twist, and Swart, 2016). Kinesio tape is recently considered as a new method, used in clinical practice and athletic performance to reduce the risk of injury and to treat musculoskeletal disorders, and injuries (Torres, Trindade, and Gonçalves, 2016). A Kinesio tape is made from cotton fabric with a viscosity that allows ventilation and water resistance. It can be stretched up to $150 \%$ of its original size (like human skin) (Oliveira et al., 2016). The application of Kinesio tape on the skin creates a tactile sensation that activates cutaneous mechanoreceptors, which might help to alleviate the pain sensation through the gatecontrol theory pioneered by Melzack and Wall (Simon S, Yeung, 2015). It's widely used to reduce pain (Jongbloed, van Twist, and Swart, 2016), increase the range of motion (Jongbloed, van Twist, and Swart 2016), inhibit or facilitate muscle, reduce inflammation (Torres, Trindade, and Gonçalves, 2016), provide joint support (Chang et al., 2010), improve lymphatic drainage (Torres, Trindade, and Gonçalves, 2016), improve walking patterns and improve functional activities (Kumbrink, 2012).

The American Rheumatology Association has proposed the use of Kinesio tape in knee osteoarthritis patients. This method is economical, though adequate evidence about the effectiveness of any particular method has not been stated (Mutlu et al., 2017). Furthermore, the results of previous studies on knee Kinesio tape in patients with knee osteoarthritis have been contradictory to some extent (Mutlu et al., 2017). Therefore, according to the studies conducted during the past, we tried to find a new method of applying Kinesio tape on a knee, which is capable of mitigating the pain of these patients, thereby enhancing their quality of life. The aim of this study was to compare the effect of Kinesio tape of the quadriceps muscle and the knee with a sham Kinesio tape on the parameters of pain and quality of life in patients above 45 years old with knee osteoarthritis. This has not been performed in previous studies as a long-term solution along with treatment and follow-up. In this study, the long-term effects of KT on knee pain and quality of life were assessed in subjects with knee osteoarthritis, which has not been evaluated earlier. Also, the combined method of applying KT used in the present study was not used in previous studies.

Previous studies have used the quadriceps muscle facilitation technique (Sarallahi et al., 2016), (Jongbloed, van Twist, and Swart, 2016), but in this study, in addition to the facilitation technique, the ligaments method was used, mentioned in the $\mathrm{K}$ - taping book (Kumbrink, 2012).

\section{Materials and Methods}

This study was a double blind, randomized clinical trial registered in the Iranian Registry of Clinical Trials: (IRCT20180916041051N1). Forty seven patients with knee osteoarthritis above 45 years-old were recruited. The diagnosis of knee osteoarthritis (tibiofemoral) was performed based on symptoms and radiological findings carried out by a specialist.

Those subjects with knee osteoarthritis (tibiofemoral) with Kellgren-Lawrence Grade II and III with the 
radiological signs in the affected side who had a knee pain history for six months or more and the extent of their pain was 4-7 according to the visual analog scale (VAS) were included, while those with the following signs and symptoms were excluded:

Knee joint injections within the previous 6 months, being subject to electrical stimulation within the previous 3 months, history of lower limbs fracture within the previous 6 months, articular inflammatory diseases, history of skin sensitivity, existence of pathology (in the lumbar, pelvic and hip), history of damage to soft structures around the knee (meniscus and ligament), patellofemoral pain syndrome on the affected side, history of knee or hip joint replacement, rheumatoid arthritis, neurological diseases, deformity or contracture in the ankle, knee or hip joints, and osteoarthritis in ankle and hip joints All participants gave written informed consent prior to participating in the study. Approval for the research was received from the ethics committee of the Iran University of Medical Sciences (IR.IUMS.REC.1397.059).

For patients who had bilateral osteoarthritis of the knee, the more affected side was examined. The studied variables included pain (during rest and activity) and quality of life. In order to measure the intensity of pain during rest and activity, the visual analog scale was used. Pain during activity was the pain that the patient felt after $10 \mathrm{~m}$ of walking (Jongbloed, van Twist, and Swart, 2016). To evaluate the quality of life, the Persian version of Western Ontario and McMaster Universities Osteoarthritis Index (WOMAC) questionnaire was used (Ebrahimzadeh et al., 2014). The questionnaire was filled before starting treatment and after the follow-up session. This questionnaire consists of three sections of 24 items (related to pain, joint stiffness, and physical functioning), self-completed by the patient (Ebrahimzadeh et al., 2014).

In the next stage, the patients were randomly assigned to intervention and control groups. Randomization was done using sealed envelopes. This study was performed as double-blind, in which the patients and the outcome assessor were blinded to the allocation. The treatment and assessment of patients were performed by two therapists. The patients were requested to stop analgesic consumption during the study (figure 1). Patients in both groups received 3 daily therapeutic exercises (three times a day with 10 repetitions every day). The exercises included: quadriceps muscle setting, straight leg raise (SLR), and active flexion-extension of the knee on a chair.

The methods of applying Kinesio tape in the treatment group are as follows: the first technique was used for rectus femoris muscle facilitation (figure 2 part A). Y shape with short sequences was used. To apply the Kinesio tape, the muscle was set in a stretched position (the patient leaned on the side, the hip was brought to extension passively, and the knee was brought to $60^{\circ}$ of flexion). The an I-shaped part of Kinesio tape was applied on the rectus femoris from the beginning of the muscle towards its end, and the Y-shaped part was applied to the upper edge of the patella with $25 \%$ of tension (two ends of the Kinesio tape without tension). The second technique was the ligament technique, with the aim of improving stability in the knee. For its application, the tape was cut along the Collateral ligaments of the knee (medial collateral ligament from the medial condyle of the femur up to Pesanserinus muscles and the lateral Collateral ligament from the lateral condyle up to head of the fibula). The tape was applied in the neutral position of the joint. Each of the tape bands was attached from the center of the joint line and with maximum tension. The ends however, were attached with attention while the knee was in maximum flexion position (figure 2 part B) (Kumbrink, 2012).

The method of applying Kinesio tape in the control group is as follows: in the control group, two horizontal Kinesio tapes (without tension) were used on the quadriceps muscle, where the length of each Kinesio tape was $10 \mathrm{~cm}$ and was used $10 \mathrm{~cm}$ and $20 \mathrm{~cm}$ above the patella (figure 2 part C) (Wageck et al., 2016). After an initial assessment, the patients underwent five sessions (two sessions per week) of Kinesio tape treatment. Also, they were revisited for follow-up one week after the termination of the treatment. 


\section{Data Analysis}

The Kolmogorov-Smirnov test was used to evaluate the normality of the distribution of the variables. A normal distribution was observed for variables in both groups. To compare, changes in the pain intensity during rest, 30 minutes after Kinesio tape, three days after the last Kinesio tape, and during followup (one week after the third assessment), before and after the treatment in both groups, repeated measures ANOVA was used. To investigate the long-term effects of Kinesio tape on the quality of life of patients, paired T-test was used.

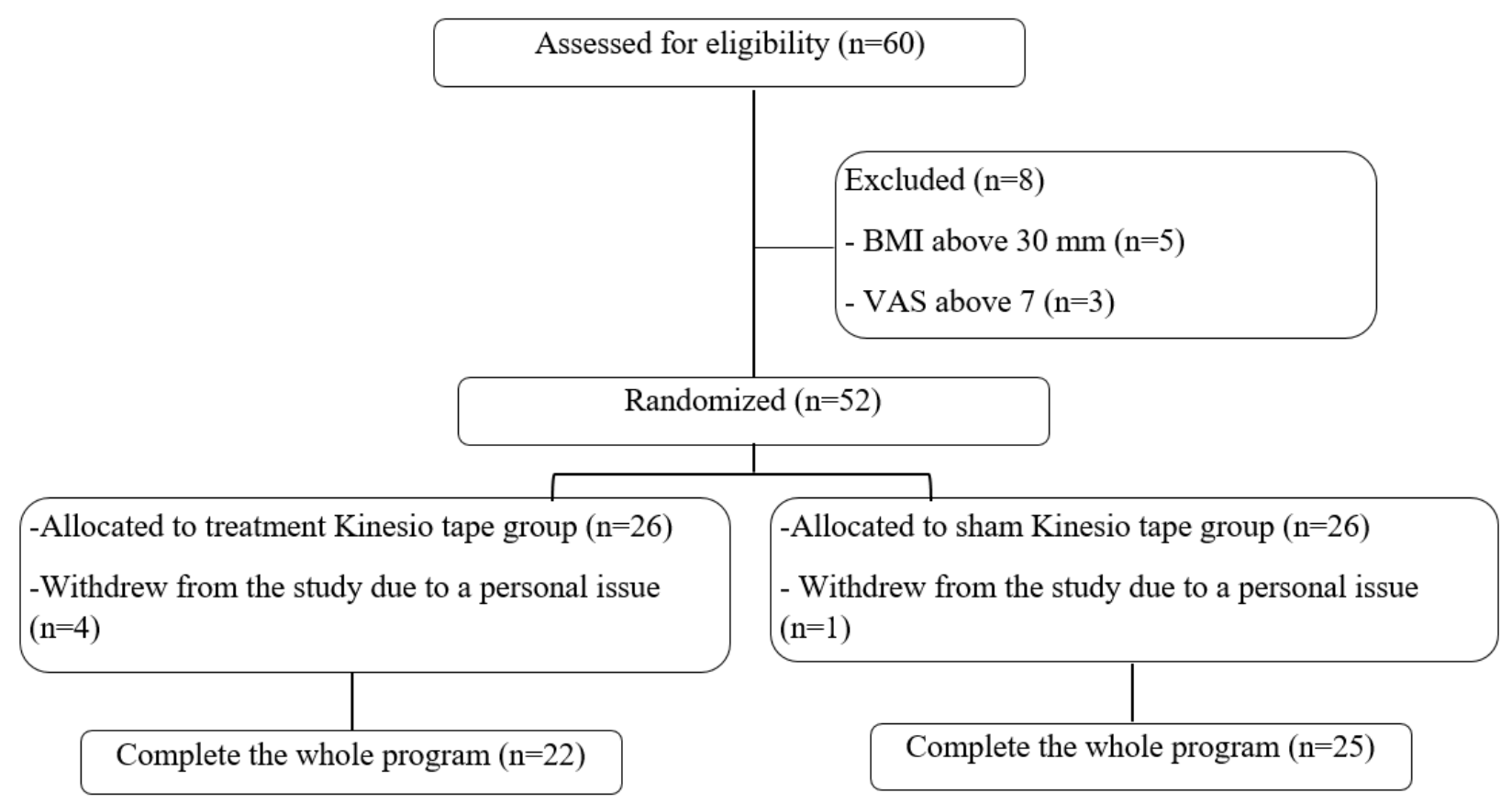

Figure 1. A flowchart of eligibility assessment through the study

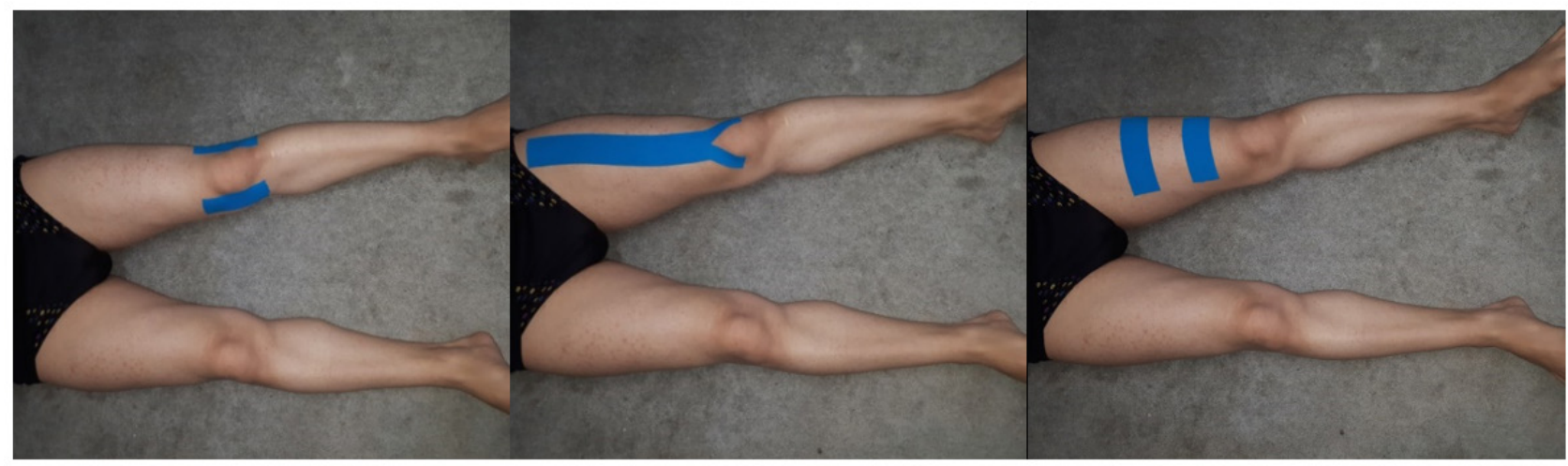

A

B

$\mathrm{C}$

Figure 2. A) Method of applying ligament Kinesio tape around the knee

B) Method of facilitation of Kinesio tape of rectus femoris

C) Method of applying the Sham Kinesio tape 


\section{Results}

The demographic characteristics of patients are shown in table 1 . The results showed that there was no significant difference between the groups in terms of height, weight, and body mass index (BMI). A total of 47 patients ( 37 women, 10 men) with the mean age of 63 years and mean BMI of 27 participated in this study.

The results of repeated measures ANOVA in withingroups showed that the knee Kinesio tape and sham tape resulted in significant reduction in pain intensity during rest and activity; both in the short-term (30 $\mathrm{min}$ after Kinesio tape) and in the long-term (five sessions after Kinesio tape) $(P=0.002)$. However, the results of repeated measures ANOVA in between-groups showed no significant pain intensity differences during the rest and during the activity between groups $(P>0.05)$ (table 2 and table 3$)$. The results of paired t-test indicated that in the long-term, knee Kinesio tape had a significant effect on improving the quality of life in both groups $(P=0.001)$. Nevertheless, there was no significant difference between groups in terms of mean changes in quality of life $(P>0.05)$ (table 4$)$.

Table 1. Descriptive statistics of the subjects

\begin{tabular}{cccc} 
variable & group & mean & P-value \\
\hline Age & treatment & $63.95 \pm 7.81$ & 0.9 \\
Year & control & $6.58 \pm 62.56$ & 0.99 \\
Height & treatment & $6.93 \pm 164$ & 0.8 \\
Cm & control & $7.43 \pm 163.92$ & 0.7 \\
Weight & treatment & $10.54 \pm 69.71$ & 0.93 \\
Kg & control & $9.74 \pm 74.92$ & 0.96 \\
BMI & treatment & $2.97 \pm 26.05$ & 0.81 \\
\hline
\end{tabular}

Table 2. Comparison of the differences in pain intensity during rest and activity in tape group for four measurement times.

\begin{tabular}{|c|c|c|c|c|c|c|c|}
\hline \multicolumn{2}{|c|}{ Variable } & $\mathbf{M} \pm \mathbf{S D}$ & $\mathbf{F}$ & P-value & $\begin{array}{c}\text { Pairwise } \\
\text { Comparisons }\end{array}$ & Mean difference & P-value \\
\hline \multirow{4}{*}{$\begin{array}{l}\text { Pain during } \\
\text { rest }\end{array}$} & Time 1 & $2.64 \pm 2.31$ & \multirow{4}{*}{19.478} & \multirow{4}{*}{$<0.001$} & Time 1 - Time 2 & $0.591(0.127-1.055)$ & 0.008 \\
\hline & Time 2 & $2.34 \pm 2.24$ & & & Time 1 - Time 3 & $1.477(0.605-2.350)$ & $<0.001$ \\
\hline & Time 3 & $1.962 .18 \pm$ & & & Time 2 -Time 3 & $0.886(0.684-1.998)$ & 0.006 \\
\hline & Time 4 & $1.882 .19 \pm$ & & & Time 1 -Time 4 & $1.750(0.212-1.561)$ & $<0.001$ \\
\hline \multirow{4}{*}{$\begin{array}{l}\text { Pain during } \\
\text { activity }\end{array}$} & Time 1 & $6.181 .71 \pm$ & \multirow{4}{*}{41.021} & \multirow{4}{*}{$<0.001$} & Time 1 - Time 2 & $0.682(0.727-2.773)$ & 0.001 \\
\hline & Time 2 & $5.581 .97 \pm$ & & & Time 1 - Time 3 & $2.023(1.305-2.740)$ & $<0.001$ \\
\hline & Time 3 & $4.722 .14 \pm$ & & & Time 2 -Time 3 & $1.341(0.684-1.998)$ & $<0.001$ \\
\hline & Time 4 & $4.722 .17 \pm$ & & & Time 1 - Time 4 & $2.432(1.500-3.364)$ & $<0.001$ \\
\hline
\end{tabular}

Time 1; primitive, Time 2; 30 minute after first Kinesio tape, Time 3; After 5 sessions Kinesio tape, \& Time 4; Follow-up. P-value* for within-subject effects test. P-value** for pairwise comparisons test. 
Table 3. Comparison of the differences in pain intensity during rest and activity in Sham-tape group for four measurement times.

\begin{tabular}{|c|c|c|c|c|c|c|c|}
\hline \multicolumn{2}{|c|}{ Variable } & $\mathbf{M} \pm \mathbf{S D}$ & $\mathbf{F}$ & P-value ${ }^{*}$ & $\begin{array}{c}\text { Pairwise } \\
\text { Comparisons }\end{array}$ & Mean difference & P-value ${ }^{* * *}$ \\
\hline \multirow{4}{*}{$\begin{array}{l}\text { Pain } \\
\text { during } \\
\text { rest }\end{array}$} & Time 1 & $3.25 \pm 2.69$ & \multirow{4}{*}{22.704} & \multirow{4}{*}{$<0.001$} & Time 1 - Time 2 & $0.300(0.038-0.562)$ & 0.019 \\
\hline & Time 2 & $2.652 .39 \pm$ & & & Time 1 - Time 3 & $0.680(0.321-1.039)$ & $<0.001$ \\
\hline & Time 3 & $1.77 \pm 1.77$ & & & Time 2 - Time 3 & $0.380(0.173-0.587)$ & $<0.001$ \\
\hline & Time 4 & $1.50 \pm 1.81$ & & & Time 1 - Time 4 & $0.760(0.314-1.206)$ & $<0.001$ \\
\hline \multirow{4}{*}{$\begin{array}{c}\text { Pain } \\
\text { during } \\
\text { activity }\end{array}$} & Time 1 & $6.201 .45 \pm$ & \multirow{4}{*}{24.098} & \multirow{4}{*}{$<0.001$} & Time 1 - Time 2 & $0.600(0.118-1.082)$ & 0.009 \\
\hline & Time 2 & $5.521 .45 \pm$ & & & Time 1 - Time 3 & $1.460(0.725-2.195)$ & $<0.001$ \\
\hline & Time 3 & $4.181 .57 \pm$ & & & Time 2 - Time 3 & $0.886(0.684-1.998)$ & 0.002 \\
\hline & Time 4 & $3.771 .88 \pm$ & & & Time 1 - Time 4 & $1.460(0.716-2.204)$ & $<0.001$ \\
\hline
\end{tabular}

Time 1; primitive, Time 2; 30 minute after first Kinesio tape, Time 3; After 5 sessions Kinesio tape, \& Time 4; Follow-up. P-value* for within-subject effects test. P-value** for pairwise comparisons test.

Table 4. Comparison of the differences in quality of life before Kinesio tape intervention, 10 days after the last Kinesio tape intervention.

\begin{tabular}{ccc} 
Variable & Mean \pm SD & P-value \\
\hline Quality of life before treatment & $42.57 \pm 14.09$ & 0.001 \\
\hline Quality of life at the end of study & 28.3812 .59 & \\
\hline
\end{tabular}

\section{Discussion}

In our study, although after therapeutic interventions, pain during rest showed a trend of improvement, no significant difference was observed between the treatment and control groups. One week after the end of Kinesio tape therapy, pain during rest in the therapeutic group was still decreasing, but no significant difference was observed between the groups. The reason for the reduction of pain in both groups can be attributed to the stimulation of sensory receptors of the skin, in turn arriving to the pain gate control theory. Pain reduction in both treatment and sham groups, can be the result of possible psychological effects (Ö, K, and M, 2016)(Williams et al., 2012). The results of a similar study conducted in 2015 (Jongbloed, van Twist, and Swart, 2016) also showed mitigation of pain in both groups, where the pain reduction was greater in the treatment group, but again no significant difference was observed between the two groups. The reasons for pain mitigation in Jongbloed study included the tension induced by Kinesio tape to the quadriceps muscle, which can reduce pain by improving muscular function and nervous feedbacks. Further, by elevating skin, Kinesio tape can improve blood circulation in the region, thereby mitigating painIt was considered as the main cause, which is a result of the pain gate theory developed by stimulating fast efferent receptors to type I However, in a study in 2016 (Mutlu et al., 2017), improvement of pain during rest was greater in the Kinesio tape group compared to the placebo group, and the difference was statistically significant. Mutlu study examined the effect of Kine- 
sio tape on pain, function, and knee range of motion. In Mutlu study, Kinesio tape was applied Y-shaped, as a facilitator on the quadriceps muscles and hamstring. The reason for this difference is due to the different methods of Kinesio tape in that study. In this study, every patient received Kinesio tape three times within 3-4 day intervals and was followed up and assessed after one month.

In this study, despite the improving trend of pain during activity, no significant difference was observed between the groups. This mean of pain mitigation did not show any descending trend within one-week follow-up, and after stopping Kinesio tape in both groups. These results were congruent with the findings of Kocyigit (Kocyigit et al., 2015), in which pain changes were not observed during activity between treatment and control groups. Nevertheless, the results of another study (Sarallahi et al., 2016) showed that Kinesio tape in the long-term is influential for pain mitigation, and a significant difference was observed between the two groups. In Sarallahi study, both groups in addition to Kinesio tape received routine physiotherapy. The results of another study (Jongbloed, van Twist, and Swart, 2016) showed that after applying Kinesio tape, pain diminished significantly during activity in the Kinesio tape group compared to the control group. In Jongbloed study, both groups received both Kinesio tape and routine physiotherapy. Jongbloed study used different therapeutic methods compared to the present study. The results of Jongbloed study showed significant pain improvement during activity following the application of Kinesio tape in the Kinesio tape group compared to the control group. In Jongbloed study, the short-term effect of Kinesio tape has been examined. Also, in Jongbloed study, the subjects who had pain for more than one year and their pain was above 5 (VAS) were explored, which can be the reason for different results demonstrated in the current study. In another study (Mutlu et al., 2017) in 2014, pain mitigation in the treatment group was statistically significant compared to the placebo group. The measurements in Mutlu study were performed once following Kinesio tape and another time after a onemonth follow-up. The reason for the discrepancy be- tween Mutlu study and the present study can be due to a different implementation of Kinesio tape and the different times of assessment. Furthermore, the mentioned studies were single-blind, hence, probability of bias exists in them.

In the Mutlu study, the quality of life was examined by the WOMAC questionnaire. The results showed a descending trend with a reduction of the mean (improve quality of life) following therapeutic interventions, but no significant difference was observed between the two groups. These results are similar to the findings of Wageck (Wageck et al., 2016). In both studies, the WOMAC questionnaire has been used, but the result did not show any significant difference between the groups. Other studies (Anandkumar, Sudarshan, and Nagpal, 2014) reported different results. Anandkumar study used the WOMAC questionnaire to investigate the quality of life and reported a significant difference between the two groups. In Anandkumar study, only women population were investigated and both groups received routine physiotherapy. In another study (Kocyigit et al., 2015), following treatment by Kinesio tape, improved quality of life was observed in both groups, but the improvement was greater in the control group. In Kocyigit study, Nottingham Health Profile (NHP) was used to investigate the quality of life. The method of implementing Kinesio tape in Kocyigit study was different from the method used in the current study.

\section{Conclusion}

Based on the results obtained from this study, it can be concluded that Kinesio tape either therapeutically or as sham can cause diminished pain and improved quality of life in patients with knee osteoarthritis. Therefore, presumably the psychological effect of the sham Kinesio tape on pain and on the quality of life of patients is as much as the therapeutic Kinesio tape method.

\section{Limitations}

In this study, subjects above 45 years of age with moderate pain intensity were examined. Therefore, it is recommended that in future studies, the therapeutic effects of Kinesio tape be explored on the pain and 
quality of life of those with younger ages and with higher pain intensities. Also, the psychological effects of Kinesio tape were not investigated, which are recommended to be examined in future studies.

\section{Acknowledgement}

The Iran University of Medical Science funded this study. The authors wish to thank all subjects who kindly participated in this research.

\section{References}

Anandkumar, S., Sudarshan, S., \& Nagpal, P. (2014). Efficacy of kinesio taping on isokinetic quadriceps torque in knee osteoarthritis: a double blinded randomized controlled study. Physiotherapy theory and practice, 30(6), 375-383.

Chang, H. Y., Chou, K. Y., Lin, J. J., Lin, C. F., \& Wang, C. H. (2010). Immediate effect of forearm Kinesio taping on maximal grip strength and force sense in healthy collegiate athletes. Physical Therapy in Sport, 11(4), 122-127. Ebrahimzadeh, M. H., Makhmalbaf, H., Birjandinejad, A., Keshtan, F. G., Hoseini, H. A., \& Mazloumi, S. M. (2014). The Western Ontario and McMaster Universities Osteoarthritis Index (WOMAC) in persian speaking patients with knee osteoarthritis. Archives of bone and joint surgery, 2(1), 57-62.

Jongbloed., Lisa., Daniëlle van Twist., and Nynke Swart. (2016). Kinesio Taping Improves Pain, Range of Motion, and Proprioception in Older Patients With Knee Osteoarthritis. American journal of physical medicine \& rehabilitation / Association of Academic Physiatrists, 95(1), e7.

Kocyigit, F., Turkmen, M. B., Acar, M., Guldane, N., Kose, T., Kuyucu, E., \& Erdil, M. (2015). Kinesio taping or sham taping in knee osteoarthritis? A randomized, double-blind, sham-controlled trial. Complementary therapies in clinical practice, 21(4), 262-267.

Kumbrink, B., \& Taping, K. (2012). An Illustrated Guide-Basics--Techniques--Indications.

Mutlu, E. K., Mustafaoglu, R., Birinci, T., \& Ozdincler, A. R. (2017). Does Kinesio taping of the knee improve pain and functionality in patients with knee osteoarthritis?: a randomized controlled clinical trial. American journal of physical medicine \& rehabilitation, 96(1), 25-33.

\section{Conflict of Interest Statement}

All the authors declare that they have no conflict of interest.
Öztürk, G., Külcü, D. G., Mesci, N., Şilte, A. D., \& Aydog, E. (2016). Efficacy of kinesio tape application on pain and muscle strength in patients with myofascial pain syndrome: a placebo-controlled trial. Journal of physical therapy science, 28(4), 1074-1079.

Oliveira, A. K., Borges, D. T., Lins, C. A., Cavalcanti, R. L., Macedo, L. B., \& Brasileiro, J. S. (2016). Immediate effects of Kinesio Taping ${ }^{\circledR}$ on neuromuscular performance of quadriceps and balance in individuals submitted to anterior cruciate ligament reconstruction: a randomized clinical trial. Journal of science and medicine in sport, 19(1), 2-6.

Roder, D. M. (2002). The epidemiology of gastric cancer. Gastric cancer, 5(1), 5-11.

Sarallahi, M., Amiri, A., Sarafzadeh, J., \& Jamshidi, A. A. (2016). The effect of quadriceps kinesio tape on functional disability, pain, and knee joint position sense in knee osteoarthritis patients. Journal of Clinical Physiotherapy Research, 1(2), 73-78.

Yeung, S. S., Yeung, E. W., Sakunkaruna, Y., Mingsoongnern, S., Hung, W. Y., Fan, Y. L., \& Iao, H. C. (2015). Acute effects of kinesio taping on knee extensor peak torque and electromyographic activity after exhaustive isometric knee extension in healthy young adults. Clinical journal of sport medicine, 25(3), 284-290.

Torres, R., Trindade, R., \& Gonçalves, R. S. (2016). The effect of kinesiology tape on knee proprioception in healthy subjects. Journal of bodywork and movement therapies, 20(4), 857-862.

Wageck, B., Nunes, G. S., Bohlen, N. B., Santos, G. M., $\&$ de Noronha, M. (2016). Kinesio Taping does not improve the symptoms or function of older people with knee osteoarthritis: a randomised trial. Journal of physiothera- 
35. The Effect of Kinesio Tape on Knee Pain and Quality of Life

py, 62(3), 153-158.

Williams, S., Whatman, C., Hume, P. A., \& Sheerin, K.

(2012). Kinesio taping in treatment and prevention of sports injuries. Sports medicine, 42(2), 153-164. 


$$
\text { مقالة يزوهشى }
$$

\section{تاثير كينزيوتيڤٍ بر درد و كيفيت زندكى در افراد مبتلا به استئو آرتريت زانو:}

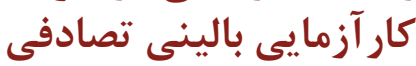

\section{على كرمى تبار'، شبنم شاهعلى"'، مهدى دادكو'، سهيل منصور سوهانى'، ارسلان قربان يور '، هاشم ابريشم كارزاده"،}

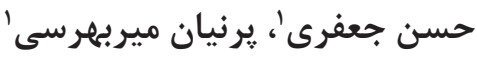

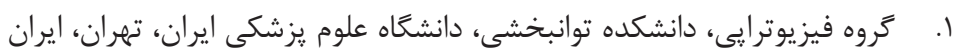

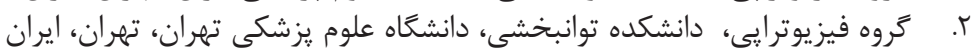

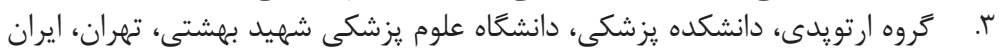

\begin{tabular}{|c|c|}
\hline جكيده & اطلاعات مقاله \\
\hline 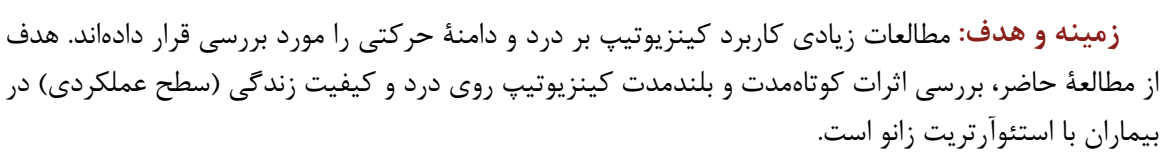 & 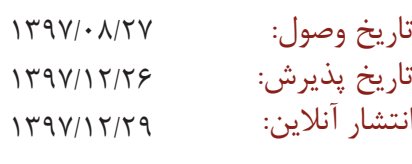 \\
\hline 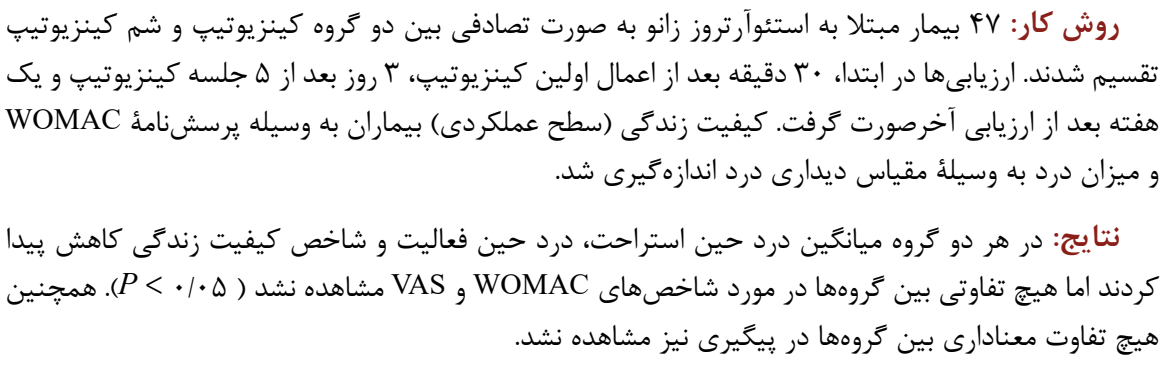 & 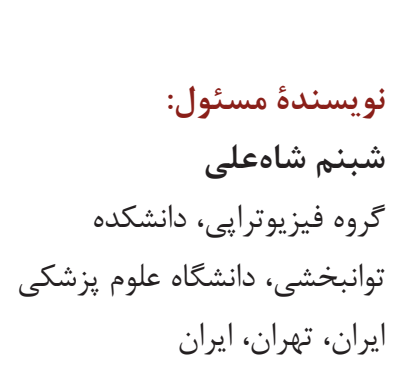 \\
\hline زانو برترى ندارد. & shabnamshahali@yahoo.com \\
\hline
\end{tabular}

